\title{
8.2 Personalmanagement und digitale Transformation
}

\section{Bibliothekarische Arbeit im Wandel}

In ihrer Doppelrolle als Kulturinstitutionen und Serviceeinrichtungen haben Bibliotheken eine lange Tradition, die immer wieder Veränderungen ihres Selbstverständnisses unterlag und keineswegs immer nur eine Geschichte von gedruckten Büchern und Zeitschriften war. Grund dafür ist, dass Charakter und Qualität der Sammlungen wesentlich vom jeweiligen Auftrag der Bibliotheken abhingen und von daher zu verschiedenen Ausprägungen bibliothekarischer Arbeit geführt haben. Schon im Übergang von der Klosterbibliothek zur Fürstenbibliothek wandelten sich die Zweckbestimmungen von Bibliotheken und damit auch das Berufsbild der Bibliothekarinnen und Bibliothekare. Die Produktion gedruckter Medien im 20. Jahrhundert hat Bibliotheken dann in neue und bis dahin unbekannte Dimensionen einer ,Versorgungseinrichtung، geführt. Zugleich ist das Bild von Bibliotheken ganz wesentlich durch gedruckte Bücher und Zeitschriften geprägt; dies ist nicht zuletzt damit zu erklären, dass Druckwerke seit dem späten 19. Jahrhundert einen Öffentlichkeits- und Verbreitungsgrad erreichten, den vorausgegangene Veröffentlichungsformen aufgrund ganz anderer Herstellungsverfahren niemals erreichen konnten. Daran wird deutlich, dass Ausrichtung und Profil von Bibliotheken unmittelbar mit der Entwicklung der Medien und mit der Geschichte der Generierung, Verbreitung und Zugänglichkeit von Wissensgütern in Zusammenhang stehen. Denn die jeweils genutzten Medien sind für genau diese Prozesse der Wissenszirkulation sehr bestimmend. Gemeinsam ist den verschiedenen Phasen der Bibliotheksgeschichte, dass es bei den ,Büchern` bzw. den Einzelstücken der Sammlungen bisher um physisch greifbare Gegenstände (,items`) ging und insofern bis zur digitalen Transformation stets analoge Medien die bibliothekarische Arbeit bestimmten. Umgekehrt bedeutet dies, dass die bibliothekarische Arbeit einer Neuorientierung unterliegt, wenn die Informationslogistik des Analogen durch Verfahren ersetzt wird, die für nicht-analoge, digitale Medien konstituierend sind.

\section{Wandel des Berufsbildes}

Hat sich mit elektronisch verfügbaren Publikationen der Auftrag von Bibliotheken grundsätzlich verändert? Für Bibliotheken steht die Versorgung mit Informationen und Literatur weiterhin im Mittelpunkt. Allerdings hat die digitale Transformation die Anforderungen und Aufgaben zur Erfüllung dieses Versorgungsauftrags deutlich 
gewandelt. ${ }^{1}$ Zudem wurden und werden weiterhin neue und zusätzliche Services entwickelt wie Informationskompetenz, Open-Access-Publizieren, Retro-Digitalisierung, Forschungsdatenmanagement, virtuelle Forschungsumgebungen etc. Auch sind neue Aufgaben hinzugekommen, die sich aus einem - oft mit Bibliotheksneubauten verbundenen - neuen Verständnis von Bibliotheken als Kommunikationszentren und Lernorten entwickelt haben; dazu gehören insbesondere Betreuung und Gestaltung von Lernräumen, Event- und Veranstaltungsmanagement sowie neue Zielgruppen. Insgesamt ist festzustellen, dass das bibliothekarische Kerngeschäft der Beschaffung, der Bereitstellung und der langfristigen Verfügbarkeit von Fachinformation und Literatur - unter veränderten medialen Bedingungen - weiterhin eine zentrale Rolle spielt und zugleich durch Servicefelder erweitert wird, die die Kernaufgaben signifikant ergänzen. Inwieweit neue Dienste, die stärker Forschungs- und Publikationsprozesse unterstützen, sich zu Routinediensten entwickeln, hängt wesentlich davon $\mathrm{ab}$, in welcher Weise sich die digitale Transformation auf den künftigen Charakter wissenschaftlicher Publikationen auswirken wird. ${ }^{2}$

Dass sich das herkömmliche Berufsbild von Bibliothekarinnen und Bibliothekaren durch den Einfluss digitaler Medien gewandelt hat, ist offensichtlich. Hinzu kommen weitere Faktoren, die mit einem veränderten Dienstleistungsverständnis öffentlich-rechtlicher Serviceeinrichtungen in Zusammenhang stehen und zumindest nicht unmittelbar auf modernen Informations- und Medientechnologien beruhen.

\section{Kunden und Nutzer}

Ein sehr deutlicher Indikator für diesen Veränderungsprozess ist die gewandelte Bezeichnung der Zielgruppen von Bibliotheken, die sich vom ,Leser‘ zum ,Nutzer“ und ,Kunden“ entwickelt hat; dies vollzog sich unter dem Einfluss von „new public management“-Ansätzen in den öffentlichen Verwaltungen Deutschlands ${ }^{3}$ und durch die Business-Orientierung von Kultur- und Wissenschaftsinstitutionen im United Kingdom und in den USA. Ohne dass diese Begrifflichkeiten hier im Detail diskutiert werden können: Was hat sich mit dem Kunden- und Nutzerverständnis der bibliothekarischen Zielgruppen geändert? Während der Kundenbegriff einen Marktcharakter von Bibliotheksservices nahelegt, lässt sich mit der Bezeichnung ,Nutzer“ ein Konsumentenverhalten verbinden. Die Botschaft beider Begrifflichkeiten ist darin zu sehen, dass bibliothekarische Arbeit nicht mehr als Verwaltungsvorgang, sondern vorrangig als kunden- und nutzerorientierte Dienstleistung zu verstehen und zu vermitteln ist.

$1 \mathrm{Zu}$ beiden Aspekten s. Naumann 2012.

2 Siehe Degkwitz 2012a.

3 Siehe Wimmer 1995. 
In welchem Umfang der Auftrag von Bibliotheken als Dienstleistung spürbar wurde, hängt eng mit dem Serviceverständnis zusammen, das die einzelnen Phasen der Bibliotheksgeschichte bestimmte. Heute ist das Dienstleistungsverständnis stark von betriebswirtschaftlichen Aspekten geprägt, was erneut den Kundenbegriff in den Mittelpunkt rückt. ${ }^{4}$ Auf diese Weise sind seit etwa 30 Jahren betriebswirtschaftliche Ansätze und Methoden in Bibliotheken eingeführt worden, die die bibliothekarische Arbeit organisatorisch beeinflusst und strukturiert haben: Strategiefindung, Leitbilderstellung, Kosten-/Leistungsrechnung, Performance-Messung, Balanced Scorecard, Benchmarking etc. sind die Stichworte, die diesen Wandel charakterisieren, der neben der digitalen Transformation zu Veränderungen bei der Gestaltung von Arbeitsabläufen unter Effizienzgesichtspunkten führt. Dass diese Bestrebungen an Grenzen stoßen, die in den Strukturbedingungen öffentlich-rechtlicher Organisationsformen begründet liegen, ist allerdings auch zu erkennen. Dazu gehört über das Haushalts- und Personalrecht hinaus vor allem der Tatbestand, dass Bibliotheken ausschließlich Sachziele und keinerlei Gewinnziele verfolgen.

Vor dem Hintergrund der skizzierten Entwicklungen stellen sich für das Personalmanagement in Bibliotheken folgende Anforderungen und Fragen:

- Wie wird die digitale Transformation auf der strategischen Ebene aufgegriffen und umgesetzt?

- Welche Qualifikationsprofile und Qualifizierungsmaßnahmen sind unter Einschluss veränderter Gehalts- und Vergütungsstrukturen erforderlich?

- Wie wirkt sich der Wandel auf Personalbedarfe und Personalstrukturen aus?

\section{Strategisches Management}

Die Herausforderungen der genannten Entwicklungen aufzugreifen und in die strategische Ausrichtung einer Bibliothek zu integrieren und umzusetzen, ist eine klare Führungsaufgabe, die in unmittelbarem Zusammenhang mit dem Personalmanagement steht. Dabei geht es nicht mehr nur um die pflichtgemäße Erfüllung von Verwaltungsvorgängen, sondern vielmehr um die Entwicklung von strategischen Zielen und die Schaffung von Voraussetzungen zur Umsetzung dieser Ziele. Von daher stehen Kunden- und Marktorientierung, Partizipation und Teamwork, Wettbewerbsfähigkeit und Wirtschaftlichkeit bibliothekarischer Leistungen und Serviceangebote im Mittelpunkt. Herkömmliche Administrations- und Verwaltungsverfahren sind deshalb nicht außer Kraft gesetzt, werden aber zunehmend von betriebswirtschaftlich getriebenen Formen der Aufgabenwahrnehmung überlagert. ${ }^{5}$ Dabei sind Lenkung und Steuerung notwendig, um auf eine systematische Umsetzung der Ziele hinzuwirken

4 Siehe Vogel/Cordes 2005.

5 Siehe Neubauer 2012a: 63f. 
und den Einsatz geeigneter Governance- und Kommunikationswerkzeuge zu gewährleisten. ${ }^{6}$ In diesen Kontext gehören in regelmäßigen Abständen stattfindende Zielfindungs- und Leitbildprozesse, Performance-Messungen und Umfeldanalysen sowie der Einsatz von Instrumenten zur laufenden Überprüfung der Zielerreichung (Balanced Scorecard, Benchmarking, Stärken-/Schwächenanalyse etc.) und Projekte zur Optimierung von Geschäftsgängen (Prozessmanagement). ${ }^{7}$ Wahrnehmung und Erfüllung dieser Aufgaben sind Bestandteil der Führungsverantwortung und von daher auf der Leitungsebene von Bibliotheken angesiedelt. Wesentlicher Erfolgsfaktor dabei ist, die Zielsetzungen, die sich aus der strategischen Ausrichtung ergeben, in die tägliche Arbeit zu integrieren. ${ }^{8}$ Das heißt, dass Kompetenzen und Potenziale der operativen Ebenen ausgeschöpft werden, um sie für die Umsetzung der strategischen Ziele zu nutzen. Erforderlich dafür sind geeignete Verfahren des Innovationsoder Veränderungsmanagements, wie sie z.B. mit Methoden zur Modellierung von Geschäftsprozessen zur Verfügung stehen. ${ }^{9}$ Oder die Innovationsansätze werden mit Projekten vorangetrieben, die modellhafte Lösungen für den Auf- und Ausbau neuer Services entwickeln. Dabei ist darauf zu achten, dass solche Projekte sich nicht verselbstständigen, indem sie an der Kultur der Organisation vorbeiziehen und im Ergebnis scheitern, da mögliche kulturelle Differenzen zwischen Organisation und Projekt unterschätzt worden sind. Andererseits müssen Projekte ihr Innovationspotenzial entfalten können, was auf Seiten der Organisation bzw. ihrer Akteure die Bereitschaft voraussetzt, vertraute Arbeitsabläufe oder Verfahren in Frage stellen zu lassen und gegebenenfalls Veränderungen zuzuführen. Ohne ein solches Spannungsverhältnis können Veränderungen nicht wirksam werden. ${ }^{10}$

\section{Personalentwicklung}

Darüber hinaus müssen Veränderungsprozesse von einem breiten Spektrum an Maßnahmen zur Personalentwicklung unterstützt werden. Dabei geht es zum einen um Maßnahmen, die die Mitarbeiterinnen und Mitarbeiter fordern und fördern, und um Anreize (,incentives`), die die Attraktivität des Arbeitsplatzes steigern und damit zu höherer Motivation und Leistungsbereitschaft beitragen. ${ }^{11}$ Fordernde und fördernde Maßnahmen sind:

6 Siehe Nürnberger 2012.

7 Siehe Neubauer 2012b.

8 Siehe Hendrix 2012.

9 Siehe Degkwitz/Klapper 2011.

10 Siehe Degkwitz 2012b: 171ff.

11 Haas-Betzwieser 2012; Tröger 2012. 
- Fachliche Kompetenz- und Know-How-Entwicklung: Dazu gehören insbesondere Fort- und Weiterbildungsmaßnahmen, die auf Anforderungen und Aufgaben im Rahmen des Medienwandels ausgerichtet sind.

- Soft-Skill-Entwicklung und Coaching: Damit werden vor allem das Training von Führungskräften und die Weiterentwicklung von Führungsverantwortung angesprochen. Beides ist für die Umsetzung strategischer Ziele auf dem ,corporate level' von großer Bedeutung.

- Beteiligungsverfahren und Partizipation: In diesem Kontext stehen Auf- und Ausbau von - gegebenenfalls gestuften - Strukturen zur Entscheidungsfindung und zur Information von Mitarbeiterinnen und Mitarbeitern im Mittelpunkt.

- Vorgesetzten-/Mitarbeitergespräche: Dabei geht es vorrangig um die Integration von ,corporate level' und ,individual level' im Veränderungsprozess, indem Kompetenz und Verantwortung der einzelnen Mitarbeiterin oder des einzelnen Mitarbeiters adressiert werden.

- Arbeitsplatzrotation und Hospitation tragen einerseits zur besseren Kooperation zwischen einzelnen Arbeitsbereichen bei. Andererseits wird dadurch Flexibilität im Arbeitseinsatz gefördert.

Anreize, die die Attraktivität des Arbeitsplatzes betreffen, sind vor allem:

- Gleichstellung, die im Sinne der Gleichbehandlung von Mitarbeiterinnen und Mitarbeitern eine kontinuierliche Anforderung an die Personalentwicklung und Karriereförderung ist.

- Flexible - familiengerechte - Arbeitszeiten, die individuelle Bedürfnisse für die Gestaltung des Arbeitsalltags berücksichtigen. In diesen Zusammenhang gehören auch Möglichkeiten der Telearbeit.

- Gesundheitsmanagement und Sportangebote während der Arbeitszeit sind ein wesentlicher Beitrag zur Aufwertung der Arbeitsplatzqualität.

- Leistungsorientierte Bezahlung, die einen weiteren wichtigen Anreiz bietet, um zur Steigerung der Attraktivität des Arbeitsplatzes beizutragen. Grundsätzlich umfasst der Tarifvertrag der Länder (TV-L) entsprechende Möglichkeiten.

Mit beiden Herangehensweisen geht es um Anerkennung und Wertschätzung der Mitarbeiterinnen und Mitarbeiter. Im Fall von Fortbildung und Kommunikation werden die Bereitschaft zur Übernahme von Verantwortung und Kompetenzpotenziale weiterentwickelt. Die Maßnahmen zur Steigerung der Attraktivität der Arbeitsumgebung tragen wesentlich zur Anerkennung persönlicher Bedingungen sowie zur Verbundenheit von Mitarbeiterinnen und Mitarbeitern mit ihrem Arbeitsplatz bei. In beiden Fällen steht die Entwicklung einer Arbeits- und Betriebskultur im Mittelpunkt, die Mitarbeiterinnen und Mitarbeiter als Kompetenz- und Verantwortungsträger sieht und ihre persönlichen Bedürfnisse ernst nimmt.

Für die Erweiterung fachlicher Kompetenzen und Qualifikationsprofile sind die Transformation der Medien und die Entwicklung des Informationsmarkts leitend. 
Von daher sind allgemeine, betriebswirtschaftliche Kenntnisse und IT-Kompetenz im Sinne von Informatik als Grundlagen hilfreich. Im Zusammenhang mit der Weiterentwicklung von Bibliotheken müssen jedoch der spezifische Impact des Medienwandels und dessen Auswirkungen auf die Produktion, Verbreitung, Aneignung und Verfügbarkeit digitaler Wissensgüter den Maßnahmen zur Kompetenzentwicklung und Weiterqualifizierung zugrunde liegen. Die absehbar steigende IT-Affinität bibliothekarischer Aufgaben erfordert deshalb die Bereitschaft von Mitarbeiterinnen und Mitarbeitern, sich mit den jeweils neuen Arbeitsverfahren vertraut zu machen und sich dafür zu qualifizieren. In Arbeitsbereichen, die ein besonders hohes Maß an IT-Kompetenz voraussetzen, wie beispielsweise im Rahmen der Administration komplexer Anwendungen und Systeme oder auf Innovationsfeldern, die ausdrücklich Informatikkenntnisse erfordern, werden Weiterbildungsmaßnahmen jedoch nur eingeschränkt ausreichen. Hier stellt sich die Notwendigkeit ein, technisches Personal einzusetzen und dafür gegebenenfalls entsprechende Stellenumwidmungen vorzunehmen.

\section{Personalbedarf und Personalstruktur}

Im Zuge der digitalen Transformation verändern sich auch die Anforderungen an die Bedarfe der Personalausstattung und der Personalstruktur vor folgendem Hintergrund. Die sich verändernde Informationslogistik digitaler Monographien und Zeitschriften beeinflusst die herkömmlichen Berechnungsgrundlagen des Personalbedarfs. Indem anstelle gedruckter Einzelstücke unter Preisbindung verstärkt Nutzungslizenzen auf Basis von Paketlösungen für E-Books und E-Journals im Rahmen von Beschaffungskonsortien oder von einzelnen Institutionen mit den Verlagen verhandelt werden, haben sich die bisherigen Verfahren von der Akquisition bis hin zur Bereitstellung stark verändert. Nicht nur, dass sich die Durchschnittswerte der Bearbeitungszeit pro Stück verschoben haben und die Bearbeitung digitaler Medien neue und zusätzliche Kompetenzen erfordert. Der weiterhin zunehmende Bezug von Nutzungslizenzen sowie die daran gekoppelten Erschließungs- und Bereitstellungsverfahren sind gegenüber gedruckten Materialien anders und neu zu gestalten. Da parallel $\mathrm{zu}$ den elektronischen Medien weiterhin gedruckte Medien zu bearbeiten sind, können die herkömmlichen Personalbedarfe noch nicht als überholt betrachtet werden. ${ }^{12}$ Doch vor allem auf dem Gebiet der formalen und sachlichen Erschließung sind auch bei Printmedien Veränderungen mit den Möglichkeiten IT-gestützter Verfahren zu verzeichnen; dazu gehören insbesondere die mittlerweile intensiv genutzten Möglichkeiten des ,shared cataloging ‘ und der damit verbundenen Optionen der Fremddatenübernahme. Hinzu kommen neue Aufgaben, die nicht unmittelbar an

12 Siehe Naumann/Umlauf 2002; Naumann 2003. 
traditionelle Kernkompetenzen anknüpfen. Dazu gehören das elektronische Publizieren, der Betrieb von Open-Access-Repositorien, die Retro-Digitalisierung, Informationskompetenz, RFID-Einsatz für die Automatisierung von Ausleih- und Rückgabevorgängen sowie weitere Mehrwertdienste zur Unterstützung von Forschung und Lehre. Viele dieser Innovationsfelder setzen IT-Kompetenz und oftmals eine hohe Affinität zu einzelnen Fachgebieten voraus. Die Beispiele verdeutlichen die Problematik von Berechnungen des Personalbedarfs. Für das traditionelle Kerngeschäft werden sich die herkömmlichen Parameter weiterhin zugrunde legen lassen. Im Hinblick auf neue Dienste können diese Berechnungsgrundlagen nicht oder nur sehr eingeschränkt angewandt werden.

Ähnlich wie bei den Berechnungsgrundlagen für den Personalbedarf wirft auch die Eingruppierungspraxis eine Reihe von Fragen und Problemen auf - gerade vor dem Hintergrund neuer Dienstleistungsfelder. Für traditionelle Bibliotheksaufgaben werden weiterhin die DBI-Materialien 201 (2000) ${ }^{13}$ herangezogen, die allerdings der Weiterentwicklung von bibliothekarischen Aufgaben nur sehr bedingt Rechnung tragen. Vor allem der Aufbau und Betrieb sowie die Weiterentwicklung vorrangig ITaffiner Dienste erfordern entsprechende Kompetenzen. Bei der Gewinnung geeigneten IT-Personals stehen Bibliotheken in unmittelbarem Wettbewerb mit der Privatwirtschaft, der gerade bei der Gehaltsentwicklung ganz andere Möglichkeiten zur Verfügung stehen. Auch erweist sich in diesem Zusammenhang als besonders nachteilig, dass die gehaltsbezogenen Entwicklungsmöglichkeiten für den gehobenen Dienst bis maximal Entgeltgruppe 9 TV-L stark beschränkt worden sind. Alle Bemühungen, diese Entwicklung rückgängig zu machen und die zuvor möglichen Vergütungsoptionen wieder wirksam werden zu lassen, konnten bisher nicht zum Erfolg geführt werden. Das ist dem sich abzeichnenden Bedarf an neu und zusätzlich qualifiziertem Personal in Bibliotheken sehr abträglich. Denn künftig werden Bibliotheken für die Bewältigung ihrer Aufgaben möglicherweise eher weniger, aber dafür deutlich höher qualifizierte Mitarbeiterinnen und Mitarbeiter benötigen, die dann allerdings auch angemessen einzugruppieren sind. Finanzielle Engpässe im Kultur- und Wissenschaftsbereich lassen allerdings eine solche Flexibilität oftmals auch dort nicht $\mathrm{zu}$, wo sie von den tariflichen Rahmenbedingungen her grundsätzlich möglich wäre.

\section{Zusammenfassung}

In einer Zeit, die einerseits durch umfassende Veränderungen des Serviceverständnisses von Bibliotheken und damit des Berufsbildes geprägt ist und in der sich andererseits rückläufige Budgets und verstärkt finanzielle Engpässe abzeichnen, gehört Personalmanagement zu den großen Herausforderungen, die unbedingt aufzugreifen

13 Siehe BIB 2001. 
sind, um die Weiterentwicklung von Bibliotheken voranzutreiben. Das erfordert auf der einen Seite eine strategische Ausrichtung, welche die Veränderungen adressiert, die sich aus dem Wandel der Medien und der Weiterentwicklung der Informationstechnologie ergeben, und die deren Umsetzung in Angriff nimmt; das bedeutet allerdings auch, dass Entscheidungen zu treffen sind, die traditionelle Arbeitsfelder durch neue Aufgaben und Rollen ersetzen. Auf der anderen Seite sind zur Umsetzung dieser strategischen Ziele eine konsequente Personalentwicklung und nachhaltige Maßnahmen eines Veränderungsmanagements erforderlich, das sich auch auf die Personalstruktur, die Qualifizierung und die Vergütungskriterien auswirkt. In Zeiten knapper Kassen fällt dies oft schwer. Doch Produktion, Verbreitung und dauerhafte Verfügbarkeit von digitalen Wissensgütern erfordern verlässliche Infrastrukturen, deren weiterer Auf- und Ausbau von gut qualifizierten Bibliothekarinnen und Bibliothekaren aktiv $\mathrm{zu}$ gestalten und $\mathrm{zu}$ realisieren ist. ${ }^{14}$

\section{Literatur}

Beger, Gabriele: Personalentwicklung. Neue Aufgaben und „altes“ Personal. In: Personal- und Organisationsentwicklung in Bibliotheken. Hrsg. von Andreas Degkwitz. Berlin: De Gruyter Saur 2012 (Bibliothek - Monographien zu Forschung und Praxis (BMFP) 2). S. 157-166.

Berufsverband Information Bibliothek e.V. (BIB) (Hrsg.):Arbeitsvorgänge in wissenschaftlichen Bibliotheken (AVWB). Beschreibung und Bewertung nach dem Bundes-Angestelltentarifvertrag (BAT). Unverändert. Nachdruck der 1. Aufl. der DBI-Materialien 201 des Ehem. Dt. Bibliotheksinst. Bad Honnef: Bock + Herchen 2001.

Degkwitz, Andreas (2012a): „Texte, Daten, Bilder - Wissen!“. In: BIBLIOTHEK - Forschung und Praxis 36 (2012), S. 215-219.

Degkwitz, Andreas (2012b): Drittmittelprojekte als Herausforderung an die Organisations- und Personalentwicklung. In: Personal- und Organisationsentwicklung in Bibliotheken. Hrsg. von Andreas Degkwitz. Berlin: De Gruyter Saur 2012 (Bibliothek - Monographien zu Forschung und Praxis (BMFP) 2). S.167-174.

Degkwitz, Andreas u. Frank Klapper: Prozessorientierte Hochschule. Allgemeine Aspekte und Praxisbeispiele. Hrsg. von Andreas Degkwitz u. Frank Klapper. Bad Honnef: Bock + Herchen 2011. Elektronische Version: http://www.dini.de/fileadmin/docs/Prozessorientierte_ Hochschule_2011.pdf (29.08.2014).

Haas-Betzwieser, Eva: Die Bibliothek von innen heraus entwickeln. Personal- und Organisationsentwicklung in der Staatsbibliothek zu Berlin. In: Personal- und Organisationsentwicklung in Bibliotheken. Hrsg. von Andreas Degkwitz. Berlin: De Gruyter Saur 2012 (Bibliothek Monographien zu Forschung und Praxis (BMFP) 2). S. 91-105.

Hendrix, Imma: Von alten Gewohnheiten und neuen Chancen: Veränderungsmanagement und Personalentwicklung für den Neubau. In: Personal- und Organisationsentwicklung in Bibliotheken. Hrsg. von Andreas Degkwitz. Berlin: De Gruyter Saur 2012 (Bibliothek Monographien zu Forschung und Praxis (BMFP) 2). S. 149-155.

14 Siehe Kempf 2013 und Umlauf/Gradmann 2012. 
Kempf, Klaus: Der Sammlungsgedanke im digitalen Zeitalter = L'idea della collezione nell'età digitale. Fiesole: Casalini Libri 2013 (Letture magistrali in biblioteconomia 6).

Naumann, Ulrich: Arbeitszeitmodelle. In: Erfolgreiches Management von Bibliotheken und Informationseinrichtungen. Fachratgeber für die Bibliotheksleitung und Bibliothekare. Hrsg. von Hans-Christoph Hobohm, Konrad Umlauf und Gabriele Beger. Loseblattsammlung. Hamburg: Dashöfer 2003, Abschnitt 4.4.

Naumann, Ulrich: Serviceportfolios von Bibliotheken im Umbruch: Herausforderungen an Management und Organisation. Ein Überblick zur Thematik aus betriebswirtschaftlicher Sicht. In: Personal- und Organisationsentwicklung in Bibliotheken. Hrsg. von Andreas Degkwitz. Berlin: De Gruyter Saur 2012 (Bibliothek - Monographien zu Forschung und Praxis (BMFP) 2). S. 13-44.

Naumann, Ulrich u. Konrad Umlauf: Personalbedarf. In: Erfolgreiches Management von Bibliotheken und Informationseinrichtungen. Fachratgeber für die Bibliotheksleitung und Bibliothekare. Hrsg. von Hans-Christoph Hobohm, Konrad Umlauf und Gabriele Beger. Loseblattsammlung. Hamburg: Dashöfer 2002, Abschnitt 4.3.

Neubauer, Wolfram (2012a): „Unsere Mitarbeiter sind unser größtes Kapital“: Methoden und Prozesse für ein erfolgreiches Personalmanagement an wissenschaftlichen Bibliotheken. In: Personal- und Organisationsentwicklung in Bibliotheken. Hrsg. von Andreas Degkwitz. Berlin: De Gruyter Saur 2012 (Bibliothek - Monographien zu Forschung und Praxis (BMFP) 2). S. 6380.

Neubauer, Wolfram (2012b): Fortschritt lebt von der Veränderung: Die Reorganisation einer Großbibliothek am Beispiel der Bibliothek der ETH Zürich. In: Personal- und Organisationsentwicklung in Bibliotheken. Hrsg. von Andreas Degkwitz. Berlin: De Gruyter Saur 2012 (Bibliothek Monographien zu Forschung und Praxis (BMFP) 2). S. 175-196.

Nürnberger, Dorothee: Allgemeine Herausforderungen an das Personalmanagement in Bibliotheken. In: Personal- und Organisationsentwicklung in Bibliotheken. Hrsg. von Andreas Degkwitz. Berlin: De Gruyter Saur 2012 (Bibliothek - Monographien zu Forschung und Praxis (BMFP) 2). S. 45-61.

Tröger, Beate: Personalentwicklung in der Praxis. In: Personal- und Organisationsentwicklung in Bibliotheken. Hrsg. von Andreas Degkwitz. Berlin: De Gruyter Saur 2012 (Bibliothek Monographien zu Forschung und Praxis (BMFP) 2). S. 81-89.

Umlauf, Konrad u. Stefan Gradmann (Hrsg.): Handbuch Bibliothek. Geschichte, Aufgaben, Perspektiven. Stuttgart, Weimar: Metzler 2012.

Vogel, Bernd u. Silke Cordes: Bibliotheken an Universitäten und Fachhochschulen. Organisation und Ressourcenplanung. Hannover: HIS 2005 (Hochschulplanung 179).

Wimmer, Ulla (Hrsg.): Verwaltungsreform. Bibliotheken stellen sich der Herausforderung. Berlin: Deutsches Bibliotheksinstitut 1995. 